\title{
Spezifische Ansätze im Kontext des Social Sellings
}

\subsection{Content Marketing}

Medialisierung, Digitalisierung, Individualisierung und die Peer-to-peerKommunikation zusammen mit einem Gefühl der kommunikativen Überforderung verändern die grundsätzlichen Rahmenbedingungen der Unternehmens- und Marketingkommunikation und zeigen die Grenzen der bisherigen Kommunikationsformate auf. Diese Erkenntnisse verlangen nach neuen Ansätzen in der Kommunikation. Sie erfordern den Wechsel von Push- zu mehr PullKommunikation (Uhl 2020, S. 7). Genau hier setzt das Content Marketing an. Trotz der seit einigen Jahren in der Praxis proklamierten Relevanz von Content Marketing, sucht man den Begriff „Content Marketing“ in einigen der großen deutschen Grundlagenlehrbüchern vergebens: So verzichten Homburg in den Werken „Grundlagen des Marketingmanagements“ (Homburg 2020a) und „Marketingmanagement“ (Homburg 2020b) und Bruhn in „Marketing - Grundlagen für Studium und Praxis“ (Bruhn 2019) auf die Diskussion dieses Marketingansatzes. Möglicherweise liegt das daran, dass die Grundidee so augenscheinlich ist, dass man sie nicht extra betonen muss:

Definition: Content Marketing „Unter Content-Marketing werden strategisch fundierte Maßnahmen zur Erstellung und Verbreitung von nützlichen Medieninhalten verstanden, die das Ziel haben, beim Adressaten marktrelevante Einstellungen und Verhaltensweisen im Sinne der Unternehmensziele zu verändern." (Meffert et al. 2019, S. 751)

Im Kontext des digitalen B2B-Marketings und damit auch im Rahmen des Social Sellings lässt sich Content Marketing als eine Inbound-Marketingtechnik bezeichnen, bei der beispielsweise über Webseiten oder soziale Medien nützliche Inhalte 
zur Verfügung gestellt werden, die zur Erlangung und Aufrechterhaltung von Markenvertrauen dienen. Um nun Inhalte zu schaffen, die für ein B2B-Publikum wertvoll und relevant sind, müssen die Social Seller ein Verständnis über die Informationsbedürfnisse der potenziellen Kunden und deren Kaufentscheidungsprozess entwickeln. Wertvolle Inhalte sind relevant, nützlich, überzeugend und aktuell. Content Marketing erfordert einen kulturellen Wandel vom „Verkaufen“ zum „Helfen“ (Holliman und Rowley 2014, S. 269).

Der Begriff „Content“ beschreibt jegliche Form von Inhalten, die Ton, Text, Stand- und Bewegtbild umfassen können (Kreutzer 2019, S. 6). Es lassen sich zahlreiche unterschiedliche Typen von Inhalten schaffen: Dazu gehören im einfachsten Fall eigene Beiträge in sozialen Netzwerken und auch Diskussionsbeiträge und Antworten auf Beiträge anderer. Zudem sind Blogs, Videos, downloadbare Berichte, Studien oder Whitepaper, E-Books, Pressemitteilungen, Podcasts, Newsletter, Ratgeberseiten, Präsentationen, Anleitungen oder Lexika (Wikis) nutzbar, um sich mit Inhalten zu positionieren. Sogar die Schaffung diverser Veranstaltungen und Events lassen sich als Content subsumieren.

Jedoch müssen bei der Schaffung von neuem Content stets die Relevanz und der Nutzen der Inhalte für die Zielgruppe im Fokus stehen. Die Nachfrage nach Informationen und Unterhaltung im Netz lässt nicht nach, jedoch ist das bestehende Content-Angebot schon heute weitaus größer als der tatsächliche Bedarf. Jeder neue Content, ob Webseite, YouTube-Video, Blogartikel oder Podcast zum gleichen Thema, erschweren es dem Nutzer, Entscheidungen zu treffen. Vor dem Hintergrund des bestehenden und wachsenden Angebots lässt auch die Sichtbarkeit der eigenen Inhalte stetig nach. Um nachhaltige Aufmerksamkeit bei der Zielgruppe zu generieren, muss man sich kontinuierlich die Frage stellen, inwieweit die Publikation des jeweiligen neuen Inhalts wirklich zielführend ist und welchen Mehrwert dieser bietet (Weller 2019, S. 3).

\subsection{Personal Branding}

Eine Marke definieren Burmann et al. allgemein als „Bündel aus funktionalen und nicht-funktionalen Nutzen, deren Ausgestaltung sich aus Sicht der Zielgruppen der Marke nachhaltig gegenüber konkurrierenden Angeboten differenziert" (Burmann et al. 2018, S. 14). Dabei wird das Selbstbild, das eine Marke von sich hat, als „Markenidentität“ bezeichnet. Das „Markenimage“ stellt das Fremdbild dar. Dieses Verständnis gilt nicht nur für Unternehmen- oder Produktmarken, sondern auch für Persönlichkeitsmarken (Personenmarke, Personal Brand oder Human Brand,Self Brand) (Dumont und Ots 2020, S. 119). Hierunter ist das ,zugespitzte 
Bild einer Person in den Köpfen ihrer Mitmenschen zu verstehen. Eine starke Persönlichkeitsmarke übernimmt eine Identifikations- und Differenzierungsfunktion und führt zur Präferenzbildung." (Spall und Schmidt 2019, S. 21).

Definition: Personal Branding „Personal Branding bezeichnet den Prozess, den ein Individuum durchläuft, um sich selbst $\mathrm{zu}$ profilieren und an andere $\mathrm{zu}$ vermarkten." (Jacobson 2020, S. 716)

Die Relevanz des in dieser Definition skizzierten Personal Brandings für den Vertrieb im Allgemeinen und das Social Selling im Speziellen ist offensichtlich: Besonders im B2B-Vertrieb mit persönlichem Kontakt vermarktet der Vertriebler nicht nur seine Produkte und Dienstleistungen, sondern auch sich selbst zu einem gewissen Grad an die Kunden. Deshalb ist der Aufbau einer Personenmarke im Vertrieb längst zu einem erfolgsentscheidenden Faktor geworden. Kunden treffen ihre Entscheidung häufig aufgrund des Vertrauens in die Persönlichkeit des Verkäufers. Solches Vertrauen entsteht nur dann, wenn Unternehmer und Vertriebsfachleute genau wissen, wofür sie stehen, und dies eindrucksvoll vermitteln können (Spall und Schmidt 2019, S. 168). Um Vertrauen zu schaffen sind Authentizität und Glaubwürdigkeit Voraussetzungen (Burmann et al. 2012, S. 136 ff.). Für das Social Selling sind diese sowie Kompetenzsignalisierung wichtig. Genau dabei kann Personal Branding unterstützen. Personal Branding ist ein bewusster Prozess, um die eigene Wahrnehmung bei relevanten Stakeholdern (nicht nur Kunden, sondern auch z. B. bei Mitarbeitern, Kollegen, Netzwerken, Öffentlichkeit) zu steuern (Spall und Schmidt 2019, S. 22). Diesozialen Medien sind nur ein Instrument für das Personal Branding. Die persönliche Markenbildung beruht auf einer Kombination aus der Online- und Offline-Präsentation des Selbst (Jacobson 2020, S. 716). Personal Branding ist langfristig angelegt. Nur so kann eine „Social Authority“ entstehen.

Definition: Social Authority ,Social Authority beschreibt den zielgerichteten Aufbau der Reputation in sozialen Netzwerken durch regelmäßige und werthaltige Postings und Interaktionen." (Spandl 2020, S. 20)

Allgemeingültige Regeln oder Rezepte für die Schaffung einer erfolgreichen, starken Persönlichkeitsmarke für den Vertriebskontext existieren allerdings nicht. Ausgangspunkt aller Kommunikation und Aktivitäten in den sozialen Medien ist ein klares Bild der eigenen Personenmarke. Spall und Schmidt (2019), auf deren ausführliches Werk „Personal Branding“ hiermit verwiesen wird, empfehlen folgende Vorgehensweise: 
1. Eigene Identität entwickeln

2. Profil schärfen

3. Persönlichkeitsmarke spürbar machen

Das Personal Branding findet dabei nicht nur digital statt. Die sozialen Medien sind lediglich Instrumente, die neben anderen (z. B. persönliche Homepage, Auftreten im „realen Leben“) zur eigenen Profilierung eingesetzt werden.

Die Nutzung sozialer Medien zur Selbstdarstellung, ohne zuvor ein Bewusstsein für die eigene Identität entwickelt zu haben, läuft Gefahr, Beliebigkeit auszustrahlen und als „digitaler Dampfplauderer“ wahrgenommen zu werden (Spall und Schmidt 2019, S. 134).

\subsection{Employee Advocacy (Corporate Influencer)}

Mitarbeiter erfüllen wertvolle übergreifende Funktionen für Organisationen, indem sie relevante Informationen über die Organisation mit externen Zielgruppen teilen. Mitarbeiter werden hierbei oft als vertrauenswürdige und glaubwürdige Informationsquellen über Organisationen wahrgenommen. Freiwillig von Mitarbeitern weitergegebene Informationen helfen Unternehmen, ihren Ruf zu verbessern (Kim und Rhee 2011). Da Mitarbeiter mit externen Zielgruppen sowohl über positive als auch negative Aspekte des Unternehmens in sozialen Medien und in persönlichen Gesprächen kommunizieren, ist es für Unternehmen wichtig, Beziehungen zu schaffen, die Mitarbeiter dazu ermutigen, sich positiv zu fühlen und positiv über ihr Unternehmen zu sprechen (Walden und Kingsley Westerman 2018, S. 594).

Definition:Employee Advocay „Employee Advocacy ist die freiwillige [positive] Außendarstellung oder Verteidigung eines Unternehmens, seiner Produkte oder seiner Marken durch einen Mitarbeiter (Men 2014, S. 262).

Die positive Fürsprache für den Arbeitgeber ist keine Neuigkeit. Schon seit jeher kommunizieren die Mitarbeiter in persönlichen Gesprächen im sozialen Umfeld über Beruf und Arbeitgeber. Die sozialen Medien bieten einen weiteren Kanal, der als Verstärker der persönlichen Kommunikation angesehen werden kann. Gerade deshalb ist Employee Advocacy im Rahmen des Social Sellings so relevant.

Eine für externe Personen überzeugende Wirkung entsteht selbstredend nur, wenn über den Arbeitgeber positive Botschaften verbreitet werden (und nicht etwa schlechte Bewertungen auf Kununu). Voraussetzung für ,positives Megaphoning“ 
ist eine hohe Ausprägung der Mitarbeiterzufriedenheit sowie der Beziehungsqualität, des Commitments und des Vertrauens der Mitarbeiter zum Unternehmen (Kim und Rhee 2011, S. 259 f.). Zudem sind ein funktionierender Informationsfluss (offener Austausch von Ideen und Informationen innerhalb der Organisation), die Angemessenheit des Zugangs zu Informationen, die Mitarbeiter für ihre Arbeit benötigen, sowie die Unterstützung durch die Führungskraft Einflussfaktoren zur Steigerung der Employee Advocacy (Walden und Kingsley Westerman 2018, S. 597 ff.). Sind diese Voraussetzungen geben, lohnt es sich über eine systematische Implementierung von unterstützenden Maßnahmen zur Anregung und Erleichterung der Aktivität der Mitarbeiter als „Corporate Influencer“ nachzudenken.

Technische Unterstützung bieten diverse Systeme (siehe Box/Beispiel ,SocialSelling-Amplifier"). Dabei sollen diese Systeme vor allem Unterstützung bieten und nicht die Authentizität einschränken. Sie dienen als Content Hub zum Entdecken und Teilen von freigegebenen Inhalten. Sie motivieren durch GamificationElemente (Leaderboard, interne Wettbewerbe etc.), integrieren bestehende Systeme und Netzwerke und ermöglichen Erfolgskontrollen (Sturmer 2020, S. 21 ff.).

\section{Beispiel:Technische Unterstützung mit Hilfe von Social-Selling-Amplifiern}

Die Grundidee von diesen Systemen ist die Erleichterung und Verstärkung der Reichweite der digitalen Marketingmaßnahmen im Allgemeinen und der Social-Selling-Maßnahmen im Speziellen. Dies geschieht durch die Nutzung des Einflusses von Mitarbeitern als Markenfürsprecher und deren Reichweite, Kontakte und Glaubwürdigkeit. Social-Amplifier-Systeme dienen also dem Management der Employee Advocacy. Amplifier unterstützen die Kreation von Content und die Planung von Postings. Sie benachrichtigten die relevanten Mitarbeiter über neuen Content und ermöglichen diesen, die Beiträge auf den individuellen (Social Media) Kanälen weiterzuverbreiten. Somit wird zum einen die Reichweite des Contents des Unternehmens erhöht, indem die Inhalte auch über die individuellen Netzwerke der Mitarbeiter verbreitet werden. Zum anderen bietet der vorbereitete Content den Mitarbeitern auch die Chance, sich durch interessante Beiträge selbst (als Experte) zu positionieren. Zudem lässt sich interessanter externer Content an die Mitarbeiter zurückspielen. Die meisten Programmpakete ermöglichen auch ein Controlling und die Analyse diverser Kennzahlen. Beispiel für einen Social-Selling-Amplifier sind beispielsweise Hootsuite Amplify, Dynamic Signal, EveryoneSocial, Ambassify, Bambu by sprout social u. v. a.m. 


\subsection{Influencer Marketing - Unterschiede im B2B- und B2C-Kontext}

Im Kontext des Social-Media-Marketings ist das Thema „Influencer Marketing“ zu einem der am stärksten und am kontroversesten diskutierten Themen avanciert. Das Interesse an dem professionellen Einbinden von Influencern in die Kommunikation und das Marketing von Unternehmen ist stark gestiegen (Lommatzsch 2018, S. 24). Der Begriff „Influencer“ leitet sich aus dem englischen „influence“ (Einfluss) ab und ist von der Wortbedeutung sehr unspezifisch.

Definition:Influencer Im Kontext des Marketings sind Influencer Personen, die mit ihren Meinungsäußerungen in sozialen Netzwerken, Blogs, auf Videoplattformen und in Communitys einen maßgeblichen Einfluss auf das Meinungsbild vieler Menschen ausüben. Durch Kombination von kommunikativer Aktivität in digitalen Netzwerken, Persönlichkeitsstärke, große Reichweite und spezifische Themenkompetenz wird Influencern besondere Glaubwürdigkeit zugesprochen. Alternativ spricht man von „digitalen Meinungsführern“ (Kreutzer und Land 2017, S. 211; Schach 2018, S. 31; Spall und Schmidt 2019, S. 136).

Influencer Marketing verknüpft die Elemente „Social Media Marketing“, „Content Marketing” und „Empfehlungsmarketing“ (Nirschl und Steinberg 2018, S. 7).

Definition:Influencer Marketing „Das Betreiben von Influencer Marketing ist eine strategische Vorgehensweise, die darauf abzielt, vom Einfluss und der Reichweite wichtiger Meinungsmacher und Multiplikatoren zu profitieren, indem diese eine Werbebotschaft für ein Unternehmen in sozialen Netzwerken und dem Social Web verbreiten." (Nirschl und Steinberg 2018, S. 11).

Influencer werden beim Influencer Marketing für Ihre Kommunikation vom Unternehmen bezahlt. Die Kommunikation richtet sich primär auf den Absatzmarkt aus. Als Ziele stehen direkte Absatzsteigerung, Reichweite, Aufmerksamkeit für Produktbotschaften und Markenbildung im Mittelpunkt. Teilweise scheinen Glaubwürdigkeit und Transparenz der Kommunikation relativ egal zu sein, solange diese Ziele erreicht werden. Die Evaluation des Influencer Marketings erfolgt mittels Absatz- und Reichweitenzahlen, Interaktionen, Conversions (Lommatzsch 2018, S. 25). Typisch ist hierbei der Einsatz von Rabattcodes oder Affiliate Links. Die Herausforderung für Unternehmen liegt darin, zur Marke und Zielgruppe passende, mit möglichst hoher Reichweite und hohem Vertrauen bei den Konsumenten ausgestatte Influencer zu wählen (Kleinjohann und Reinecke 2020, S. 27). 
Tab. 3.1 Abgrenzung Social Selling - Influencer Marketing

\begin{tabular}{l|l|l}
\hline Kriterium & Social Selling & Influencer Marketing \\
\hline Zeitliche Perspektive & Grundsätzlich langfristig & Sehr kurzfristige Ziele möglich \\
\hline Kommunikation & $\begin{array}{l}\text { Zielt am Ende auf individuelle } \\
\text { Kommunikation (one-to-one) }\end{array}$ & $\begin{array}{l}\text { Massenkommunikation mit } \\
\text { Followern }\end{array}$ \\
\hline Einsatzschwerpunkt & B2B & B2C \\
\hline Grundidee & $\begin{array}{l}\text { Aufbau eines eigenen } \\
\text { (Beziehungs-) Netzwerks }\end{array}$ & $\begin{array}{l}\text { Nutzen des bestehenden } \\
\text { Netzwerks Dritter }\end{array}$ \\
\hline Herausforderung & $\begin{array}{l}\text { Schaffung werthaltigen } \\
\text { Contents und regelmäßige } \\
\text { Aktivität }\end{array}$ & $\begin{array}{l}\text { Finden passender Influencer und } \\
\text { Erhaltung der Glaubwürdigkeit }\end{array}$ \\
\hline Kostenentstehung & $\begin{array}{l}\text { Zeitaufwand für Pflege des } \\
\text { Netzwerks }\end{array}$ & $\begin{array}{l}\text { Direkte Bezahlung } \\
\text { Erfolgskontrolle }\end{array}$ \\
Nur bedingte Zurechenbarkeit & $\begin{array}{l}\text { Direkte Zurechenbarkeit } \\
\text { (Rabattcodes, Affiliate Links, } \\
\text { Tracking, Sondereditionen etc.) }\end{array}$ \\
\hline
\end{tabular}

Zumeist wird das Influencer Marketing im B2C-Kontext verstanden. In vielen Bereichen ähnelt es dem Social Selling und man könnte Social Selling auch als B2B-Influencer Marketing sehen. Jedoch unterscheiden sich beide in vielen Aspekten (vgl. Tab. 3.1).

\subsection{Social Listening - Social Media Monitoring}

Social Media Monitoring ist der eher passive Aspekt im Kontext des Social Sellings und dient vor allem der Informationssammlung. Es zielt auf die Beschaffung von Wissen über Kundeninteressen und -bedürfnissen, zur Wahrnehmung des eigenen Images und des Images der Wettbewerber sowie aktuelle Entwicklungen und Trends.

Social Media Monitoring versucht, auf Basis der realen Kommunikation in sozialen Netzwerken Erkenntnisse über die Wahrnehmung der Leistungen, Produkte, Marken, Hashtags, Personen etc. des eigenen Unternehmens sowie der Wettbewerber zu erhalten. Bei diesen „Informationen über Informationen“ gilt es, besondere Häufungen von Lob und Reklamationen, geäußerte Erwartungshaltungen, konkrete Produktanregungen oder auch Trends aus der Vielzahl der Meinungsäußerungen in den sozialen Medien herauszudestillieren. Dabei geht es im Kern darum, den Online-Nutzern möglichst genau zuzuhören (Kreutzer 2019, 
S. 5 f.; Kreutzer et al. 2020, S. 59; Newberry 2019). Auch wird ermittelt, wie intensiv über Themen und Trends gesprochen wird sowie in welchen Umfeldern und in welchen Kontexten dies erfolgt (Kreutzer 2018a, S. 86). Das Monitoring erfolgt permanent mittels automatisierten Suchen (über Webcrawler, Searchbots) im (Social) Web nach definierten Kriterien (z. B. Keywords, Themen, Kontexten, Sprachen, Regionen) (Liu 2016, S. 186).

In der wissenschaftsorientierten Fachliteratur wird „Social Listening“ als Synonym zum Social Media Monitoring gesehen und alternativ auch „Buzz-Tracking“ genannt (Kreutzer 2018a, S. 85-86; Kreutzer et al. 2020, S. 59). Laut Praktikern ist Social Listening aktiver, da es aus den Erkenntnissen des Monitorings zukunftsgerichtete Aktivitäten und direkte Maßnahmen zu entwickeln versucht (Beckam 2020; Newberry 2017). Diese Ansätze beziehen sich allerdings eher auf Massenkommunikation in B2C-Anwendungsfällen. Bei Social Selling im B2BKontext verschafft sich der Vertriebler durchs Zuhören in den sozialen Medien sehr gute Ansatzpunkte zur individuellen Kontaktaufnahme, ohne in aggressiver Manier mit der Tür ins Haus zu fallen.

Zahlreiche Angebote von Monitoring Tools ermöglichen die (teil-) automatisierte Überwachung bzw. das Monitoring der Aktivitäten in den sozialen Netzwerken (z. B. Talkwalker, Storyclash, reputation.com, sprout social, critical mention, hootsuite u. v. m).

Open Access Dieses Kapitel wird unter der Creative Commons Namensnennung 4.0 International Lizenz (http://creativecommons.org/licenses/by/4.0/deed.de) veröffentlicht, welche die Nutzung, Vervielfältigung, Bearbeitung, Verbreitung und Wiedergabe in jeglichem Medium und Format erlaubt, sofern Sie den/die ursprünglichen Autor(en) und die Quelle ordnungsgemäß nennen, einen Link zur Creative Commons Lizenz beifügen und angeben, ob Änderungen vorgenommen wurden.

Die in diesem Kapitel enthaltenen Bilder und sonstiges Drittmaterial unterliegen ebenfalls der genannten Creative Commons Lizenz, sofern sich aus der Abbildungslegende nichts anderes ergibt. Sofern das betreffende Material nicht unter der genannten Creative Commons Lizenz steht und die betreffende Handlung nicht nach gesetzlichen Vorschriften erlaubt ist, ist für die oben aufgeführten Weiterverwendungen des Materials die Einwilligung des jeweiligen Rechteinhabers einzuholen.

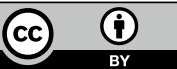

